Pneumologie
Kompass Pneumol 2021;9:334-337

DOl: $10.1159 / 000519980$

\title{
Spektrum Pneumologie - wissenswert, kompakt, anregend
}

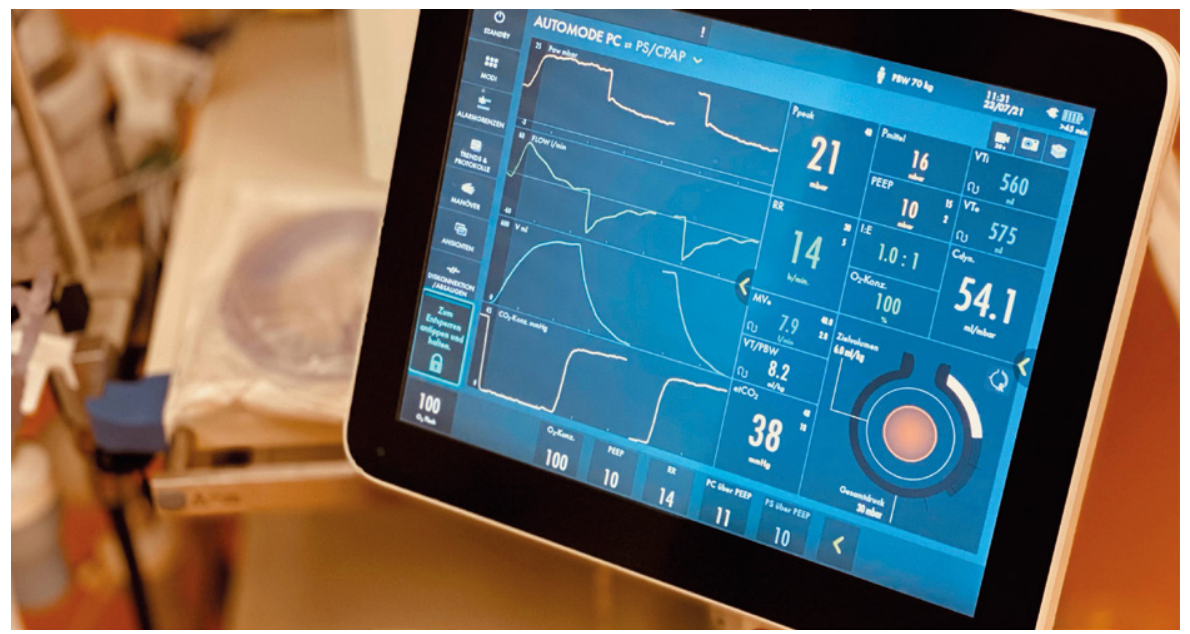

Philipps-Universität Marburg

\section{Krebsmedikament schürt Hoffnung für schwere COVID-19-Fälle}

Vor einem Jahr hatte ein Team um den Marburger Mediziner Prof. Dr. Andreas Neubauer bereits vom Erfolg einer Ruxolitinib-Verabreichung bei einer schwer erkrankten COVID-19-Patientin berichtet, die künstlich beatmet wurde. Das Medikament Ruxolitinib stammt ursprünglich aus der Krebstherapie: Das Mittel hemmt Enzyme im Körper, die an überschießenden Entzündungsreaktionen beteiligt sind. Da eine überschießende Immunantwort oft mit erhöhter Sterblichkeit bei einer COVID-19-Erkrankung einhergeht, untersuchte das Team um Neubauer, ob eine Verabreichung von Ruxolitinib regelmäßig das Überleben von künstlich beatmeten Erkrankten verlängert. Das Team aus Marburg und Kassel schloss in seine Studie 16 künstlich beatmete COVID-19-Patientinnen und -Patienten im Alter von 35-92 Jahren ein, die allermeisten davon waren Männer. Sie erhielten das Medikament für 4-28 Tage, zusätzlich zur Stan- dardbehandlung, die z.B. die Verabreichung des entzündungshemmenden Medikaments Dexamethason umfasst.

«Im Vergleich mit anderen publizierten Behandlungen schneidet die zusätzliche Ruxolitinib-Verabreichung gut ab», erklärt Dr. Caroline Rolfes von der Klinik für Anästhesiologie, Intensivmedizin, Notfallmedizin und Schmerztherapie am Klinikum Kassel: Die Überlebensrate lag bei $81 \%$. In früheren Studien lag diese am Tag 28 zwischen 25-60\%. Noch eine weitere Beobachtung teilt das Team mit: Diejenigen Patientinnen und Patienten, die bis zum Ende künstlich beatmet werden mussten, waren bereits vor Studienbeginn mehr als einen Tag lang auf eine Beatmungsmaschine angewiesen, also bevor sie das Medikament erhielten. «Der Beginn der Ruxolitinibbehandlung hat sich für das Ergebnis als kritisch erwiesen», schlussfolgert Mitverfasser Dr. Thomas Wiesmann von der Marburger Klinik für Anästhesie und Intensivtherapie.
Beatmungsgeräte sind unerlässlich für die Behandlung schwerer Fälle von COVID-19, jetzt kommt eine medikamentöse Unterstützung in Sicht. Foto: Dr. Thomas Wiesmann
«Natürlich war die Anzahl der Patientinnen und Patienten, die wir in unsere Studie eingeschlossen haben, zu klein, um endgültige Aussagen über die Wirksamkeit des Medikaments bei COVID-19 zu treffen», erklärt Neubauer. Außerdem verzichtete das Team auf eine Kontrollgruppe, um niemandem die Behandlung mit Ruxolitinib vorzuenthalten. Mittlerweile läuft bereits eine gröBere klinische Studie, um weiter zu untermauern, ob Ruxolitinib den COVID-19-Erkrankten mit schwerem Krankheitsverlauf einen Vorteil bringt.

\section{Literatur}

1 Neubauer A, et al.: Leukemia. 2021;35(10):2917-2923. 
Institut für Qualität und Wirtschaftlichkeit im Gesundheitswesen (IQWiG) FOKUS

\section{Nivolumab + Ipilimumab: Zusatznutzen bei Pleuramesotheliom mit nicht epitheloider Tumorhistologie}

Das maligne Pleuramesotheliom ist ein aggressiver Tumor des Brustfells, der um die Lunge herum wächst und in die umliegenden Gewebe und Organe wie den Herzbeutel und die Lunge eindringen kann. Häufig bildet er auch Metastasen in den örtlichen Lymphknoten. Ausgelöst wird er zumeist durch eine Asbest-Exposition, die Jahrzehnte zurückliegen kann. Die Prognose ist für Frauen besser als für die häufiger betroffenen Männer, und sie hängt zudem von der Tumorhistologie ab: Menschen mit einem epitheloiden Mesotheliom überleben im Mittel länger als solche mit einem nicht epitheloiden Mesotheliom, an dem andere Zelltypen beteiligt sind.

Solange nur eine Seite betroffen ist, kann man die Lungenhälfte einschließlich der Pleura entfernen. Oft wird das Pleuramesotheliom allerdings für eine erfolgreiche Resektion zu spät diagnostiziert. In solchen Fällen wurde bislang häufig eine Strahlentherapie oder eine Kombination aus Pemetrexed und einer platinhaltigen Chemotherapie zur Behandlung eingesetzt.

Seit einigen Jahren deuten Studien darauf hin, dass auch bestimmte monoklonale Antikörper die Lebensspanne verlängern, wenn sie auch das Tumorwachstum nicht dauerhaft stoppen können. Mit Nivolumab + Ipilimumab wurde nun erstmals eine Wirkstoffkombination aus derSubstanzklasse der Immuntherapeutika für die Erstlinientherapie zugelassen. Der Gemeinsame Bundesausschuss (G-BA) hat eine Therapie nach ärztlicher Maßgabe als zweckmäßige Vergleichstherapie festgelegt. Laut Leitlinien kommen die Zweierkombinationen Pemetrexed + Cisplatin und Pemetrexed + Carboplatin sowie die Dreierkombination Bevacizumab + Cisplatin + Pemetrexed in Betracht. Der Hersteller legt in seinem Dossier Daten aus einer randomisierten kontrollierten Zulassungsstudie vor, in deren Vergleichsarm lediglich die beiden Zweierkombinationen eingesetzt wurden. Über die Vor- und Nachteile von Nivolumab + Ipilimumab für Patienten, die auch mit Bevacizumab + Cisplatin + Pemetrexed hätten behandelt werden können, sind daher keine Aussagen möglich; ein Zusatznutzen gegenüber dieser Dreierkombination ist damit nicht belegt.

Von den Personen, die an der Zulassungsstudie teilnahmen, hatten $78 \%$ ein epitheloides und 22\% ein nicht epitheloides Pleuramesotheliom. Insgesamt überlebten die Teilnehmerinnen und Teilnehmer im Median mit der neuen Wirkstoffkombination etwa 4 Monate länger als unter Pemetrexed und einer platinhaltigen Chemotherapie. Allerdings zeigte sich hier eine starke Effektmodifikation durch die Tumorhistologie: Während das mediane Gesamtüberleben beim epitheloiden Subtyp im Interventionsarm knapp 19 Monate und im Vergleichsarm gut 16 Monate betrug, ist der Unterschied beim nicht epitheloiden Subtyp mit knapp 17 gegenüber knapp 9 Monaten statistisch signifikant und klinisch relevant: Für den prognostisch nachteiligen Subtyp wurden hier mediane Überlebensspannen in derselben Größenordnung erreicht wie bislang nur für den prognostisch vorteilhaften Subtyp.

Auch in der Kategorie Nebenwirkungen gibt es Effektmodifikationen durch die Tumorhistologie: Bei einem epitheloiden Mesotheliom traten unter der neuen Wirkstoffkombination mehr schwerwiegende unerwünschte Ereignisse und mehr Erkrankungen der Nieren und Harnwege auf als unter der zweckmäßigen Vergleichstherapie, während beim nicht epitheloiden Subtyp jeweils keine statistisch signifikanten Unterschiede zu beobachten waren. Entsprechend zweigeteilt fällt das Bewertungsergebnis aus: Für Betroffene mit nicht epitheloider Tumorhistologie gibt es einen Hinweis auf einen beträchtlichen Zusatznutzen. Bei epitheloider Tumorhistologie ist ein Zusatznutzen von Nivolumab + Ipilimumab im Vergleich zu einer Kombination aus Pemetrexed und einer platinhaltigen Chemotherapie nicht belegt.

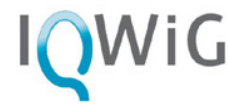

www.iqwig.de

Friedrich-Alexander-Universität Nürnberg-Erlangen

\section{Kann ein Chemokin eine schützende Rolle bei Asthma bronchiale einnehmen?}

Asthma bronchiale ist eine chronisch-entzündliche Erkrankung der Atemwege. Bei den entzündlichen Vorgängen spielt die Ansammlung von Zellen der Immunabwehr, den eosinophilen Granulozyten, in den Atemwegen eine wichtige Rolle. Während eine bestimmte Form der eosinophilen Granulozyten, abgekürzt iEos, entzündliche Prozesse fördert, trägt eine zweite Form, rEos genannt, zum Gleichgewicht in der Lunge bei. Die Forschungsgruppe um Prof. Dr. Dr. Susetta Finotto und Doktorandin Nina Li von der Abteilung Molekulare Pneumologie verabreichten Mäusen mit und ohne Asthma eine künstlich hergestellte Form des
Chemokins RANTES. Am Universitätsklinikum Erlangen beobachtete, dass sich als Folge die Zahl der rEos erhöhte, während die iEos abnahmen. Daneben wurde vermehrt der gegen Entzündungen wirkende körpereigene Stoff IL-10 hergestellt, was auf eine möglicherweise antientzündliche Wirkung von RANTES hindeutet. Außerdem zeigten die Forschenden, dass das Asthma-Medikament Resiquimod die RANTES-Produktion bei Kindern mit Asthma einleitet, was ebenfalls für eine unterstützende Wirkung des Chemokins bei der Behandlung der Erkrankung spricht. Das Forschungsteam stellte zudem fest, dass bei einer Infektion mit Rhino- viren, die Schnupfen und Erkältung auslösen, die Produktion von RANTES in den Blutzellen von Kindern mit Asthma bronchiale verringert war. Rhinovirus-Infektionen führen häufig zu Verschlechterungen von Asthma. Mit ihren Ergebnissen sowie Auswertungen der Daten der USA-AsthmaBRIDGE-Kohorte und von Blutuntersuchungen von Kindern mit Asthma eröffnet die FAU-Forschungsgruppe eine neue Sichtweise auf RANTES in der Therapie von Asthma bronchiale.

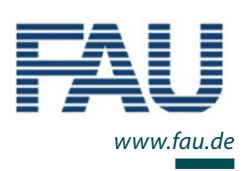




\section{Wilhelm Sander-Stiftung FOKUS}

\section{Kleines Molekül steuert Lungenkrebs: Forschungsgruppe der TU Darmstadt entschlüsselt Mechanismus der Tumorkommunikation}

Das Funktionieren eines mehrzelligen Organismus erfordert die genaue Koordination aller beteiligten Zellen - in gesundem Gewebe ebenso wie in Tumoren. Die Kommunikation zwischen den Zellen ist dabei von wesentlicher Bedeutung und erfolgt über direkten zellulären Kontaktoder über Botenstoffe. Bei der Kommunikation innerhalb der Zelle spielen besonders die in Exosomen enthaltenen mikroRNA (miRNA) eine wichtige Rolle. MikroRNA sind kleine Ribonukleinsäure-Moleküle, die eine zentrale Rolle bei der Regulation der Genexpression und der zellulären Proteinsynthese spielen.

In einem von der Wilhelm Sander-Stiftung geförderten Forschungsprojekt hat die Arbeitsgruppe um Dr. Meike Saul, Fachbereich Biologie der TU Darmstadt, die physiologische Funktion von solchen exosomalen miRNA untersucht und konnte zuletzt mit ihren Untersuchungen zum Lungenkrebs einen großen Erfolg erzielen. Lungenkrebs ist weltweit die führende Ursache für krebsbedingte Todesfälle. Das nicht kleinzellige Lungenkarzinom (nonsmall cell lung cancer, NSCLC) ist dabei mit

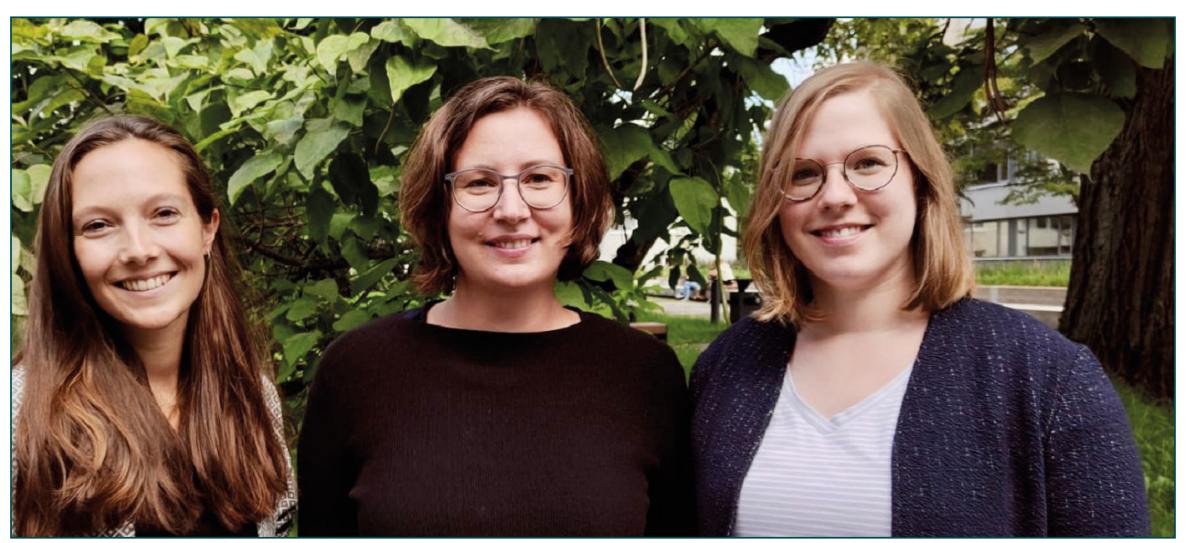

Das Forschungsteam «Extracellular vesicles \& miRNA research» des Fachbereichs Biologie der TU Darmstadt: Dr. Meike Saul mit ihren wissenschaftlichen Mitarbeiterinnen Dr. Julia Donzelli (re.) und Eva Pröstler (li.). @ Sheila Nevermann trazellulärer und exosomaler miR-574-5p steuert den PGE2-Spiegel über eine Rückkopplungsschleife. So lässt sich möglicherweise auch das Tumorwachstum beeinflussen.

Mit dieser Studie konnten Saul und ihre Kolleginnen erstmalig zeigen, dass die Funktion einer miRNA innerhalb der Zelle gegensätzlich zu ihrer Funktion im Exosom sein kann. Abhängig vom Aufnahmemechanismus kann eine exosomale miRNA an unterschiedlichen Stellen innerhalb einer Zelle freigesetzt werden, was die Funktion der miRNA entscheidend beeinflussen kann.

Dieser neu entdeckte Zusammenhang zwischen miR-574-5p und PGE2 eröffnet eine neue therapeutische Möglichkeit für Lungenkrebs. «Die Ergebnisse geben die Grundlage für die Entwicklung innovativer und personalisierter Therapieansätze», so Saul. «Die Kombination von Standard-Krebstherapien mit Inhibitoren der PGE2-Synthese stellt eine vielversprechende Behandlungsstrategie dar.» Zu den bekannten PGE2-Hemmern gehören zum Beispiel nicht steroidale Antirheumatika (NSAR). «Leider konnte die tumorhemmende Wirkung von PGE2-Inhibitoren nicht bei allen Tumorpatienten und -patientinnen beobachtet werden», sagt Saul. Dies sei auf die individuelle PGE2-Syntheserate der Erkrankten zurückzuführen. Ziel ist es daher, einen Biomarker zu finden, der Patientinnen und Patienten identifiziert, die von der Gabe von PGE2-Inhibitoren profitieren könnten (Stratifizierungsmarker).

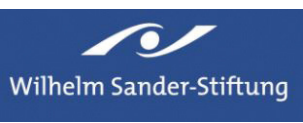

www.wilhelm-sander-stiftung.de

European Respiratory Society (ERS) und European Lung Foundation (ELF)

\section{ERS und ELF beteiligen sich am IMI-Projekt OPTIMA über künstliche Intelligenz im Gesundheitswesen}

Die European Respiratory Society (ERS) und die European Lung Foundation (ELF) sind zusammen mit 34 multidisziplinären Partnern Konsortialmitglieder des OPTIMA-Projekts der Innovative Medicines Initiative (IMI), das die Behandlung von Patienten mit Prostata-, Brust- und Lungenkrebs durch künstliche Intelligenz verbessern soll. Das Projekt ist einzigartig positioniert, um Praktiken zur Generierung von Evidenz im Gesundheitswesen zu entwickeln, um reale Evidenz in Leitlinien für die klinische Praxis (CPGs) einzubeziehen, und kann auch dazu beitragen, Best-Practice-Verfahren für die
Entwicklung von CPGs zu etablieren, die Analytik und Evidenz auf der Grundlage von KI-Modellen einbeziehen.

\section{(1) ERS ELF}

www.ersnet.org
336

Kompass Pneumol 2021;9:334-337 DOI: $10.1159 / 000519980$ 


\section{Wissenschaftlerinnen entwerfen 3D-Modell der unreifen Lunge}

Frühgeborene leiden häufig an Atemnot und müssen künstlich beatmet werden. Die physiologische und strukturelle Unreife der Lungen führt zu einer erhöhten Sterblichkeit. Eine Störung der fetalen Lungenentwicklung kann zudem schwerwiegende klinische Folgen für das Neugeborene, wie das akute Atemnotsyndrom und die chronische Lungenerkrankung Frühgeborener namens Bronchopulmonale Dysplasie, haben. Um wirksame Therapien für den zu frühen Start ins Leben zu entwickeln, nutzen Forschende aktuell

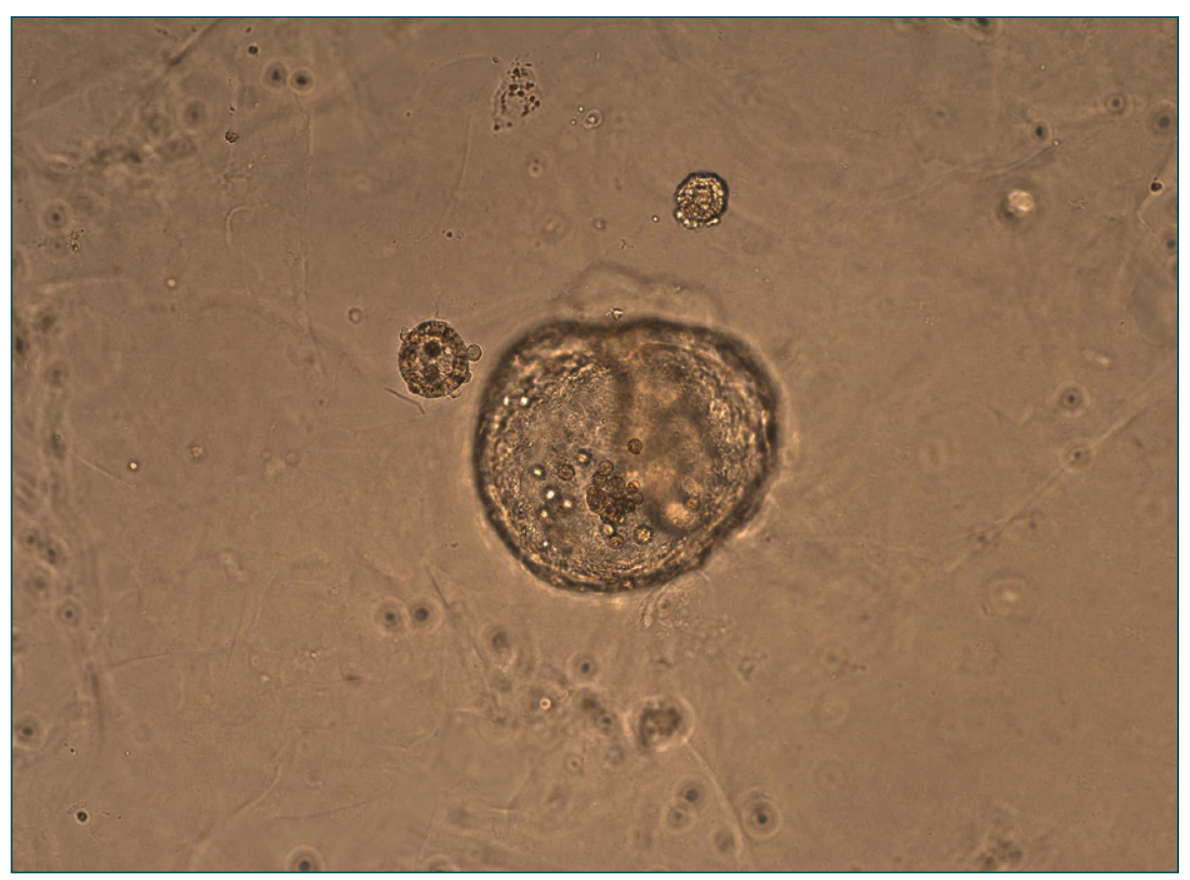

vor allem zweidimensionale Zellkulturmodelle oder testen neue Wirkstoffe im Tierversuch.

Die Leipziger Wissenschaftlerinnen Dr. Mandy Laube von der Medizinische Fakultät und Dr. Claire Fabian vom IZI haben nun ein dreidimensionales Modell der noch nicht fertig ausgebildeten Lunge entworfen. Damit können sie die Entwicklung der Lunge im zeitlichen Verlauf beobachten. Das innovative 3D-Modell soll künftig die Entwicklung neuer Therapien und Wirkstoffe erleichtern und den Bedarf

Es dauert im Labor etwa 3 Wochen, bis das blasenartige Modell der unreifen Lunge fertig ist. Foto: Dr. Mandy Laube an Tierversuchen reduzieren. Das Ergebnis ist kürzlich veröffentlicht worden.

Dr. Laube hat die ersten Lungenorganoide zunächst mit Hilfe eines Tiermodells generiert und erklärt: «Dies ermöglicht es uns, die biologischen Funktionen der Lungenorganoide mit etablierten Labormodellen, wie primären Zellkulturen und Lungenschnitten oder Tiermodellen, zu vergleichen. Zudem dienen sie uns als Basis, um im nächsten Schritt humane Lungenorganoide herzustellen.» Organoide spiegeln wesentliche Aspekte der strukturellen Gewebeorganisation sowie Organfunktion wieder und können zur Modellierung von Entwicklungs- und Krankheitsprozessen verwendet werden.

«Unsere Lungenorganoide sollen die Lunge in ihrer vorgeburtlichen Entwicklung abbilden, damit man die Organreifung untersuchen und die für das frühgeborene Kind lebenswichtigen Funktionen verbessern kann», erläutert Dr. Laube. Das Forschungsprojekt wurde mit finanzieller Unterstützung aus dem Europäischen Fonds für regionale Entwicklung durchgeführt.

\section{Literatur}

1. Laube M, et al.: Front Med (Lausanne). 2021 Sep 6;8:678438.

\section{Deutsche Gesellschaft für Pneumologie und Beatmungsmedizin (DGP) \\ Wohin bei Long- und Post-COVID? Fachgesellschaften veröffentlichen erste Patientenleitlinie}

Müdigkeit, Erschöpfung, Konzentrationsstörungen - 10-15\% der Patientinnen und Patienten klagen nach einer Infektion mit SARS-CoV-2 über mehr als 200 Symptome. Medizinerinnen und Mediziner bezeichnen diese Langzeitfolgen als Long- oder PostCOVID-Syndrom. Betroffene wissen häufig nicht, was sie tun sollen oder an wen sie sich wenden können. Die Deutsche Gesellschaft für Pneumologie und Beatmungsmedizin e.V. (DGP) hat deshalb zusammen mit anderen Fachgesellschaften und Betroffenen die neue Patientenleitlinie «Long-/Post-COVID-Syndrom» veröffentlicht, die sich speziell an Betroffene und Angehörige richtet. Die Leitlinie gibt einen Überblick über aktuelle Symptome der Erkrankung und zeigt auf, was Erkrankte tun können, um mit den Beschwerden zurechtzukommen. Bei der Leitlinie handelt es sich um eine sogenannte Living Guideline eine lebende Leitlinie, die basierend auf neuen wissenschaftlichen Erkenntnissen laufend aktualisiert wird. Sie steht auf der Website der AWMF zum Download bereit: www.awmf.org/leitlinien/detail/I//020-027. html.

Deutsche Gesellschaft für Pneumologie und Beatmungsmedizin e.V

www.pneumologie.de 